\title{
Improvement of Polysilicon Oxide Characteristics by Fluorine Incorporation
}

\author{
Horng Nan Chern, Chung Len Lee, and Tan Fu Lei
}

\begin{abstract}
The effect of fluorine on the polysilicon oxide (polyoxide) characteristics is investigated. It is found that the polyoxide leakage current and breakdown strength are improved as fluorine is incorporated into the oxide film. Experimental results show that the improvement is believed to be due to the oxide stress relaxation rather than the change of the polyoxide/polysilicon interface texture.
\end{abstract}

\section{INTRODUCTION}

$\mathbf{I}^{\mathrm{s}}$ $\mathrm{N}$ order to obtain good data retention characteristics for non-volatile memory, the inter-polysilicon oxides with a low conductivity and a high breakdown field are pursued [1]. It had been reported that the breakdown strength of the polyoxide is mainly determined by the polysilicon/polyoxide interface roughness $[2,3]$. Fluorine had been investigated to be applied to single crystal $\mathrm{Si}$ (c-Si) metal-oxide-semiconductor (MOS) device to increase the device hot-carrier immunity and irradiation hardness [4]. These reliability improvements were suspected to be due to the strain relaxation of the interface. Recently, it had been experimentally demonstrated that the c-Si oxide grown in $\mathrm{NF}_{3}$ ambient exhibited a smaller stress than those grown in dry $\mathrm{O}_{2}$ [5]. In this letter, we report that the introduction of fluorine into polyoxide reduces its leakage current and increases its breakdown strength.

\section{EXPERIMENT}

A polysilicon film of 3000 LAA thickness was deposited at $625^{\wedge}$ \{lcirc $\} C$ by a low-pressure chemical vapor deposition (LPCVD) system on p-type silicon substrate on which a 5000\} $\AA$ thick thermal oxide was grown at $1050^{\circ} \mathrm{C}$ in wet $\mathrm{O}_{2}$. The polysilicon was implanted with $5 \times 10^{15}$ arsenic/ $/ \mathrm{cm}^{2}$ at 120 $\mathrm{keV}$ and then annealed at $850^{\circ} \mathrm{C}$ for $60 \mathrm{~min}$ in an $\mathrm{N}_{2}$ gas. After defining the polysilicon island, a $270 \AA$ gate oxide was grown at $850^{\circ} \mathrm{C}$ in a dry $\mathrm{O}_{2}$ ambient. Then an LPCVD amorphous silicon of $800 \AA$ thickness was deposited at $550^{\circ} \mathrm{C}$. After defining the top-gate pattern, a $200 \AA$ pad oxide was grown at $850^{\circ} \mathrm{C}$ in dry $\mathrm{O}_{2}$ on the amorphous silicon and then implanted with $2 \times 10^{15}$ phosphorus $/ \mathrm{cm}^{2}$ at $40 \mathrm{keV}$. The dopant was annealed at $850^{\circ} \mathrm{C}$ for $60 \mathrm{~min}$ in an $\mathrm{N}_{2}$ gas. Fluorine ions with doses of $1 \times 10^{13}, 1 \times 10^{14}, 1 \times 10^{15}$ and $3 \times 10^{15} \mathrm{~cm}^{-2}$ were implanted at $25 \mathrm{keV}$ through the pad

Manuscript received November 3, 1993; revised February 23, 1994. This research was supported by the National Science Council of R.O.C. through the contract of NSC-82-0404-E009-190.

The authors are with the Department of Electronics Engineering and Institute of Electronics, National Chiao Tung University, Hsinchu, Taiwan, R.O.C.

IEEE Log Number 9401148.

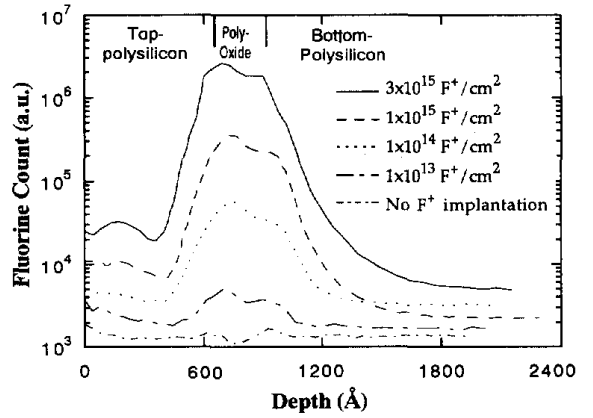

Fig. 1. The SIMS profiles of fluorine for the devices implanted with $1 \times 10^{13}$ $\mathrm{cm}^{-2}, 1 \times 10^{14} \mathrm{~cm}^{-2}, 1 \times 10^{15} \mathrm{~cm}^{-2}, 3 \times 10^{15} \mathrm{~cm}^{-2}$ and without fluorine implantation. The post-implantation annealing temperature is $850^{\circ} \mathrm{C}$.

oxide and then annealed at $850^{\circ} \mathrm{C}$ for $60 \mathrm{~min}$ in a $\mathrm{N}_{2}$ gas. For the control samples, no fluorine was implanted, but they were also subjected to the same high temperature annealing as fluorine-implanted samples. All devices were covered with a $2000 \AA$ plasma-enhanced chemical vapor deposition (PECVD) $\mathrm{SiO}_{2}$ for passivation. Contact holes were opened, and $\mathrm{Al}$ were deposited and the pattern. Finally, all devices were sintering at $350^{\circ} \mathrm{C}$ for $35 \mathrm{~min}$ in $\mathrm{N}_{2}$ gas. The I-V characteristics of the polyoxides were measured by HP 4145 semiconductor parameter analyzer.

\section{RESULTS AND DISCUSSIONS}

The SIMS profiles of fluorine for the devices implanted with $0,1 \times 10^{13}, 1 \times 10^{14}, 1 \times 10^{15}$, and $3 \times 10^{15} \mathrm{~F}^{+} / \mathrm{cm}^{2}$ followed by annealing at $850^{\circ} \mathrm{C}$ in an $\mathrm{N}_{2}$ ambient are shown in Fig. 1. It is seen that fluorine accumulated within the polyoxide and the higher the fluorine dose, the higher the fluorine peak value. There are also some amount of fluorine existing within the polysilicons. Fig. 2(a) and (b) shows the $J-E$ characteristics of the polyoxides implanted with different doses of fluorine for the positive top-gate bias (electron injection from the bottomgate) and the negative top-gate bias (electron injection from the top-gate), respectively. It is seen that all the fluorineimplanted polyoxides exhibit a smaller leakage current and a higher breakdown strength than the non-implanted polyoxide. This result is in contrast to that of previous reports on the c-Si SiO_\{2\}, i.e., fluorine did not affect or even degrade the oxide breakdown strength and its leakage current [6], [7]. In this experiment, it is found that the optimum dose of fluorine to improve the polyoxide $J-E$ characteristics is $1 \times 10^{15} \mathrm{~cm}^{-2}$. Too much fluorine seems to cause degradation to polyoxide 


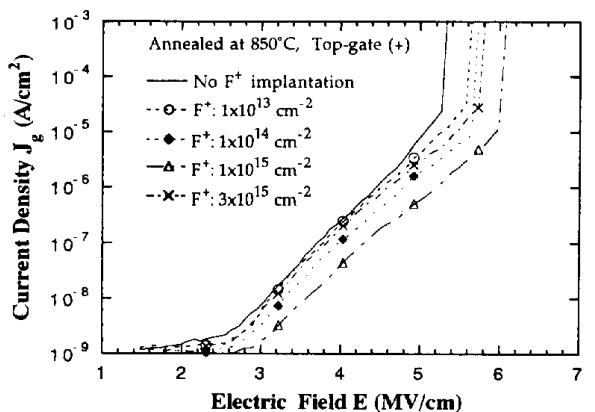

(a)

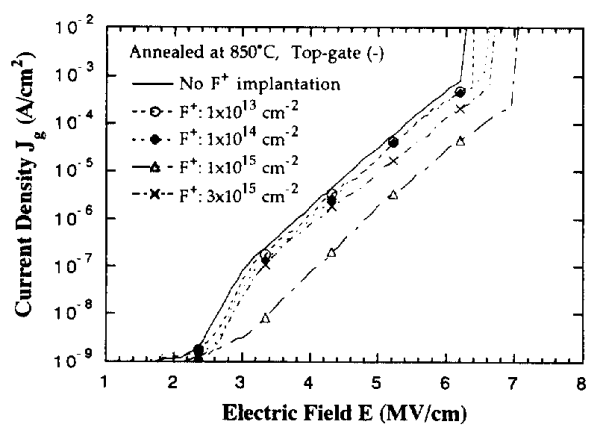

(b)

Fig. 2. The $J-E$ characteristics of the polyoxides implanted with different doses of fluorine for (a) positive top-gate bias (electron injection from the bottom gate), and (b) negative top-gate bias (electron injection from top gate).

characteristics. In Fig. 2, the less tunneling leakage current when top-gate is positively biased is attribute that the bottompolysilicon/polyoxide interface has a smoother morphology [2]. Fig. 3 shows the effective barrier height extracted from Fowler-Nordheim plots of these samples at positive top-gate bias. It is seen that the effective barrier heights were almost the same for these samples. Since the barrier height value is very sensitive to the interface texture structure [8], this implied that the fluorine-induced improvement of polyoxide characteristics was not caused by the change of the polysilicon/polyoxide interface texture. We would then suspected that the improvement of the polyoxide property is caused by the oxide stress relaxation due to the incorporation of fluorine. When fluorine was implanted into the top polysilicon and then driven in, it diffused into the polyoxide as revealed in Fig. 1. Originally, due to the high stress in the polyoxide [9], there are many strained bonds within the oxide, especially at regions nearby grain boundaries. These strained bonds are easy to be broken by high energy electrons to cause breakdown when a field is applied to the oxide. After the fluorination, the fluorine in the

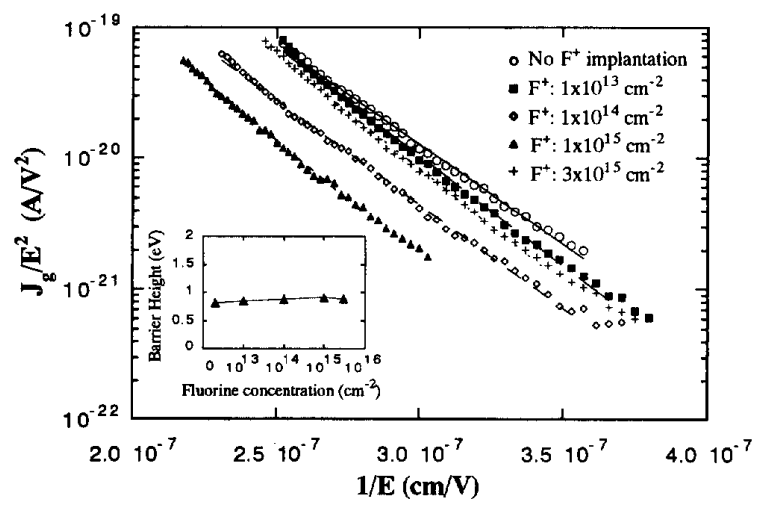

Fig. 3. Fowler-Nordheim plots for the polyoxide leakage currents at positive top-gate bias. The insert shows the effective barrier heights for the polyoxide implanted with different fluorine doses.

oxide tends to break the strained bonds and releases the oxide film stress. Hence, the oxide breakdown strength is improved.

\section{SUMMARY}

In summary, it is found that the incorporation of fluorine in the polyoxide reduced the oxide leakage current and increased the breakdown strength. This improvement is believed to be due to the oxide stress relaxation rather than the change of the polysilicon/polyoxide interface texture.

\section{REFERENCES}

[1] S. Mori, Y. Kaneko, N. Arai, Yoichi, H. Araki, K. Narita, E. Sakagemi, and K. Yoshikawa, "Reliability study of thin inter-poly dielectrics for non-volatile memory application," in Proc. 28th IRPS, pp. 132-144, 1990.

[2] S. L. Wu, T. Y. Lin, C. L. Lee, and T. F. Lei, "Electrical characteristics of textured polysilicon oxide prepared by low-temperature wafer loading and $\mathrm{N}_{2}$ preannealing process," IEEE Electron Device Lett., vol. 14, pp. 113-114, 1993.

[3] M. Hendriks and C. Mavero, "Phosphorus doped polysilicon for double poly structure," J. Electrochem. Soc., vol. 138, pp. 1466-1474, 1991.

[4] T. P. Ma, "Metal-oxide-semiconductor gate oxide reliability and the role of fluorine," J. Vac. Sci. Technol. A, vol. 10, no. 4, pp. 705-712, 1992.

[5] D. Kouvatsos, J. G. Huang, and R. J. Jaccodine, "Fluorine-enhanced oxidation of silicon: effects of fluorine on oxide stress and growth kinetics," J. Electronchem. Soc., vol. 138, pp. 1752-1755, 1991.

[6] P. J. Wright, M. Wong, and K. C. Saraswat, "The effect of fluorine on gate dielectric properties," in IEDM Tech. Dig., 1987, pp. 574-577.

[7] Y. Shioya, S. Kawamura, I. Kobayashi, M. Maeda, and K. Yanagida, "Effect of fluorine in chemical-vapor-deposited tungsten silicide film on electrical breakdown of $\mathrm{SiO}_{2}$ film," J. Appl. Phys., vol. 61, pp. 5102-5109, 1987.

[8] K. Shinada, S. Mori, and Y. Mikata, "Reduction in polysilicon oxide leakage current by annealing prior to oxidation," J. Electronchem. Soc., vol. 132, pp. 2185-2188, 1985.

[9] A. Shintani and H. Nakashima, "Anomalous stress in thermal oxide of polycrystalline Si," Appl. Phys. Lett., vol. 36, pp. 983-985, 1980. 